\title{
Single-UV-photon 2-D imaging with multi-GEM detectors
}

\author{
J.M. Maia ${ }^{\text {b,c,* }}$, D. Mörmann ${ }^{\text {a }}$, A. Breskin ${ }^{\text {a }}$, R. Chechik ${ }^{\text {a }}$, J.F.C.A. Veloso ${ }^{\text {c }}$, J.M.F. dos Santos ${ }^{\text {c }}$ \\ ${ }^{a}$ Department of Particle Physics, The Weizmann Institute of Science, 76100 Rehovot, Israel \\ ${ }^{\mathrm{b}}$ Department of Physics, University of Beira Interior, 6201-001 Covilhã, Portugal \\ ${ }^{\mathrm{c}}$ Instrumentation Center, Department of Physics, University of Coimbra, 3004-516 Coimbra, Portugal
}

Available online 18 May 2007

\begin{abstract}
The main goal being the construction of large-area, low-cost position-sensitive micropattern gaseous photomultipliers (GPMs) based on gas electron multipliers (GEM) cascades, we have studied the single-photon imaging capabilities of a 4-GEM GPM with a reflective CsI-photocathode deposited on the top surface of the 1st GEM. The charge readout was performed with a simple/economic Wedge and Strip (W\&S) readout electrode, using pulses induced through a resistive electrode. We have demonstrated the feasibility of single-photon imaging GPM with sub-millimeter resolution; single-photon spatial resolutions as small as $\sim 150 \mu \mathrm{m}$ (FWHM), i.e. $\sim 65 \mu \mathrm{m}$ (RMS), were measured in $\mathrm{Ar} / 5 \% \mathrm{CH}_{4}$ gas mixture at atmospheric pressure.
\end{abstract}

(C) 2007 Elsevier B.V. All rights reserved.

PACS: 29.40.Cs; 29.40.Gx; 29.40.-n

Keywords: Single-UV-photon imaging; Gaseous imaging detector; Gaseous photomultipliers; Gas electron multiplier; GEM; GPM

\section{Introduction}

In the last few years, substantial developments have been achieved in the field of micropattern gaseous photomultipliers (GPMs) [1], based on gas electron multipliers (GEMs) [2] combined with a solid photocathode. The photocathode can be semitransparent, deposited on a quartz window, or reflective, deposited on the top surface of the first GEM in the cascade [3]. These devices can detect single photons from the ultraviolet to the visible spectral range. The GPM working principle (Fig. 1) can be summarized as below: photoelectrons ejected from the photocathode are extracted and focussed into the first GEM holes, where they undergo multiplication; the avalanche electrons are then extracted and transported to the next GEM in the cascade, suffering further multiplication, until being extracted to an induction region and finally collected by means of a readout electrode [4].

\footnotetext{
*Corresponding author. Department of Physics, University of Beira Interior, 6201-001 Covilhã, Portugal. Tel.: + 00351275319840 ; fax: +00351275319719 .

E-mail address: jmaia@ubi.pt (J.M. Maia).
}

The low optical transparency of multi-GEM cascades (typically $\approx 12 \%$ per GEM) and the confinement of the avalanches in the holes are responsible for an efficient suppression of photon-mediated feedback effects on the photocathode and in the gas medium. Moreover, they can intrinsically reduce the ion back-flow to the photocathode, and therefore reduce its damaging and aging [5]. The signal induced in the readout element is entirely due to the collected avalanche electrons, without any slow ion component. This results in very fast (few tens of ns) detectors with high intrinsic counting-rate capability, and high time resolution (few ns) [6]. The high gains achieved with multi-GEM multipliers $\left(\approx 10^{6}\right)$ allow a high sensitivity to single electrons and, consequently, permits an efficient detection of single photons. On the other hand, the operation at atmospheric pressure allows the construction of large area, flat geometries and low-cost devices, capable of operating in high magnetic fields.

A singular characteristic of the multi-GEM structures is that the readout and multiplication stages are electrically decoupled, allowing an independent choice of the readout element pattern [7]. Typically, small pitch readout electrodes (e.g. orthogonal or small-angle strips, hexagonal pads) 


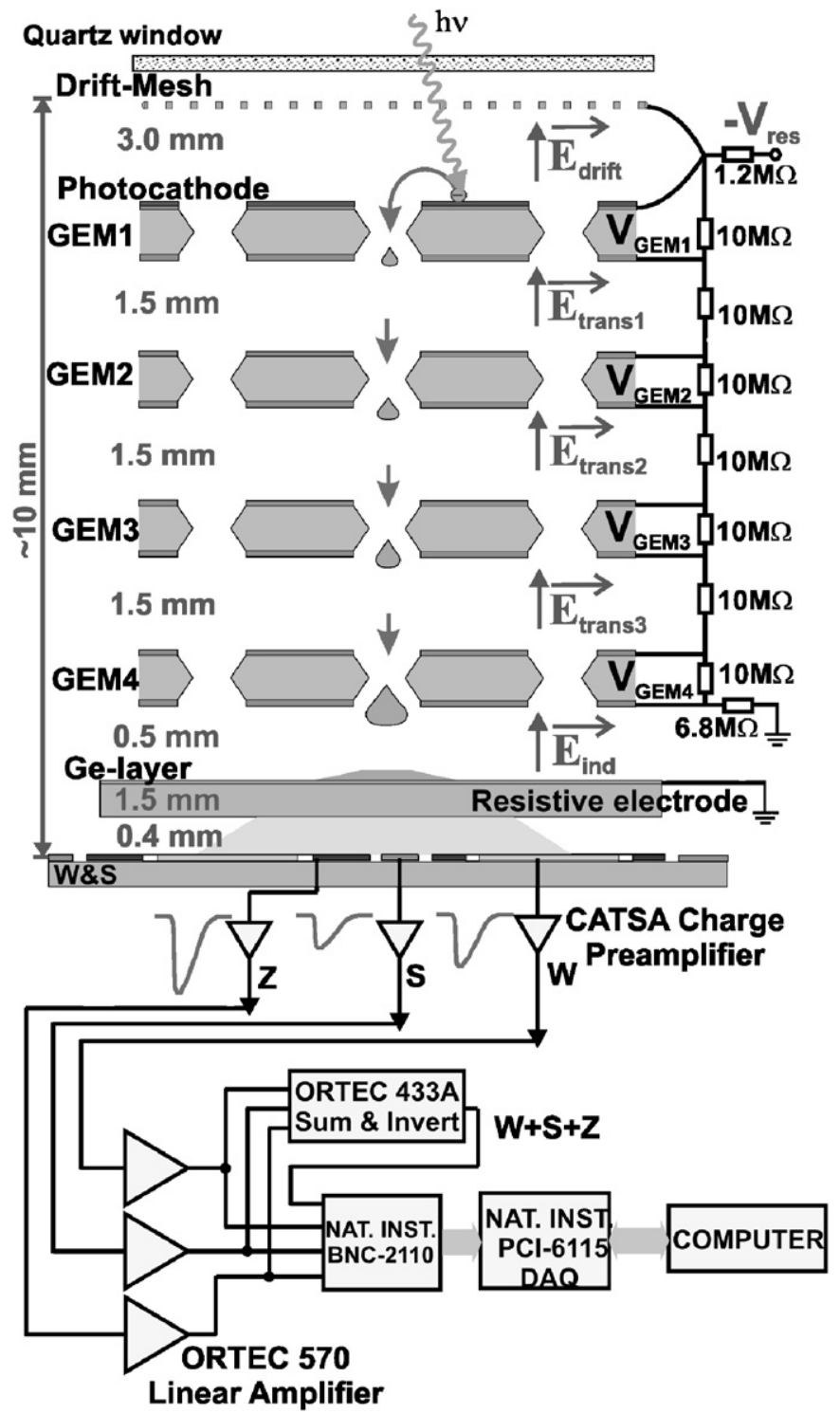

Fig. 1. Schematic view of the position-sensitive 4-GEM GPM with the block diagram of the position encoding electronics used with the W\&S readout and the biasing scheme with a resistor network.

have been used associated with highly integrated and expensive readout electronics [8]. However, readout methods based on larger pitch readout electrodes and on smaller number of electronic channels (e.g. charge-division method with resistive strips, delay-line readout with moderate pitch strips) can be an economic alternative $[9,10]$, despite its counting-rate limitations.

In this work we report the application of the Wedge and Strip (W\&S) readout technique [11] combined with a resistive electrode [12], to investigate the UVphotons imaging capabilities of multi-GEM-based GPMs. The resistive electrode, placed in front of and at a close distance from the readout electrode, is used to spread out the charge cloud to match the W\&S large pitch (Fig. 1).

\section{Experimental setup}

The position-sensitive 4-GEM GPM structure is depicted in Fig. 1. The $28 \times 28 \mathrm{~mm}^{2}$ GEMs are made from $50-\mu \mathrm{m}$ Kapton substrate coated with $5-\mu \mathrm{m}$ copper clad on both sides; they have bi-conical holes of $\varnothing 50 / 70 \mu \mathrm{m}$ in the Kapton/copper, with a density of $\sim 60 / \mathrm{mm}^{2}$ in a hexagonal lattice of $140 \mu \mathrm{m}$; the optical transparency being $\approx 12 \%$. The GEMs were mounted on 1.5-mm-thick G-10 frames. The drift-mesh is made of stainless-steel wires, $\varnothing 50 \mu \mathrm{m}$ and $500 \mu \mathrm{m}$ in pitch, having $\approx 81 \%$ optical transparency. A $\varnothing 14-\mathrm{mm}$ CsI reflective photocathode, $2500-\AA$ thick layer, was deposited by vacuum evaporation on the top-side of the 1st GEM (Au-plated with Au film $<1 \mu \mathrm{m}$ thick). The $40 \times 40 \mathrm{~mm}^{2}$ resistive electrode is made of 30 -nm-thick Gelayer vacuum deposited on a 1.5-mm-thick G-10 substrate, with a surface resistivity of $\sim 1 \mathrm{G} \Omega / \square$. As charge readout, a W\&S anode, having three electrodes, with a pitch of $1.6 \mathrm{~mm}$ and an active area of $20 \times 20 \mathrm{~mm}^{2}$, was used. The copper electrodes pattern was inscribed on a $0.6-\mathrm{mm}$-thick insulating substrate by photo-lithographic printed circuit board technology. The charge cloud induced on the readout is shared by the wedge (W), strip (S) and zigzag (Z) electrodes, and the $x$-and $y$-coordinates of an event are determined from the fraction of charge collected on $\mathrm{S}$ and $\mathrm{W}$, respectively [11].

All elements of the photodetector were mounted inside a stainless-steel gas chamber equipped with a $\varnothing 20 \mathrm{~mm}$ and 1-mm-thick quartz window. The CsI photocathode was illuminated with a continuous $\mathrm{UV} \mathrm{Hg}(\mathrm{Ar})$-lamp collimated to $\sim \varnothing 1 \mathrm{~mm}$, which produces a constant flux of photoelectrons emitted from the photocathode. Attenuators were used to reduce the photon intensity hitting the photocathode, to achieve the conditions for single-photon detection.

The photodetector was operated with an $\mathrm{Ar} / 5 \% \mathrm{CH}_{4}$ gas mixture in flow mode, at $1 \mathrm{~atm}$.

To determine the 2-D localization of events, the W\&S readout requires only three electronic channels to process the data. The 4-GEM avalanche charge induced on the $\mathrm{W}$, $\mathrm{S}$ and $\mathrm{Z}$ electrodes is integrated and pre-amplified in CATSA charge preamplifiers (sensitivity of $0.95 \mathrm{~V} / \mathrm{pC}$, rise time of $\sim 250 \mathrm{~ns})$. The charge pulses are then amplified and shaped with Ortec 570 linear amplifiers $(10-\mu$ s shapingtime constant) and added in an ORTEC 433A analog adder; the sum pulse provides the trigger to the analogto-digital converters (ADCs). The resultant pulses are further processed and analyzed by a PC-based data acquisition (DAQ) system, which is based on a National Instruments PCI-6115 board, having four simultaneously sampling analog inputs $\left(10^{7}\right.$ samples/s $)$ connected to 12-bit ADCs.

An algorithm written in LabView for Windows was used to communicate with the DAQ-board and to control the pulse sampling, digitalization, data manipulation and storage in the PC hard-disk or displaying the data on-line on a PC-screen. It allows pulse-height analysis and the 
computation in real time of the centroid position of the charge distribution recorded by the $\mathrm{W} \& \mathrm{~S}$ readout.

\section{Results and discussion}

The single-photoelectron avalanche gain distribution (Fig. 2) was recorded by irradiating a photocathode area of $\sim 150 \mathrm{~mm}^{2}$ with UV photons. The data acquired was fitted to an exponential curve [13] and an average avalanche gain of $\sim 5 \times 10^{5}$ was estimated for the photodetector.

The spatial resolution of the 4-GEM GPM for single photons was measured using a mask with two rectangular slits of $140-$ and $160-\mu \mathrm{m}$ widths, $6.5-\mathrm{mm}$ in length and $5.15-\mathrm{mm}$ apart. To determine the $x$ - and $y$-position resolutions the slits were set parallel and perpendicular, respectively, to the strips (S) of the W\&S. The projections of the single-photon images were recorded with the mask placed on the top of the drift-mesh at a distance of $\sim 3 \mathrm{~mm}$ from the photocathode, in order to minimize the divergence of the light beam on the photocathode plane; the UV lamp was placed at a distance of $\sim 25 \mathrm{~cm}$ from it. Spatial resolutions of $\sim 250$ and $\sim 150 \mu \mathrm{m}$ (FWHM) were obtained for the $x$ - and $y$-position, respectively, Fig. 3, using the 160$\mu \mathrm{m}$ width slit projections, after the correction of its width [14]. For the $\mathrm{W} \& S$ readout, the two principal factors affecting the spatial resolution are the electronic noise, which depends mainly on the inter-electrode capacitances, and the partition noise, resulting from the statistical fluctuations in the charge division between the readout electrodes [11]. The contribution of these factors to the spatial resolution depends on geometric parameters, as the fractional area of the electrode at the required position, the maximum and minimum value for the fractional area and on the total charge collected. These geometric

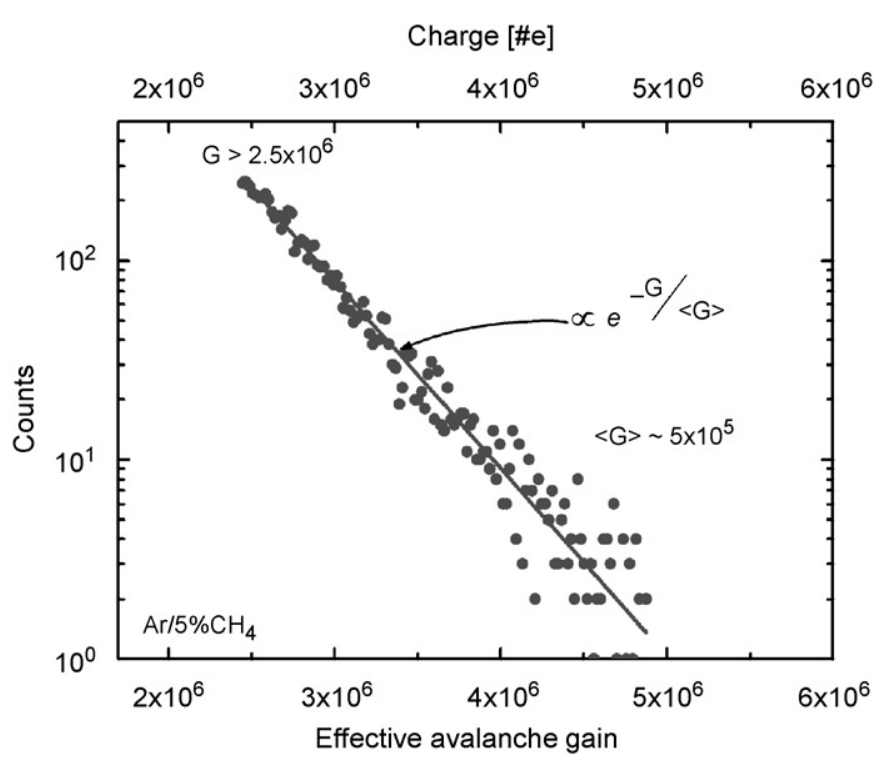

Fig. 2. Single-photoelectron gain distribution measured with the positionsensitive 4-GEM GPM for a threshold gain of $\sim 2.5 \times 10^{6}$. parameters also justify the observed dependence of the spatial resolution on $x$ - and $y$-positions (see Fig. 3).

In Fig. 4, we present a 2-D single-photon image of a metal mask with $\sim 18 \times 14 \mathrm{~mm}^{2}$, in which the letters etched have a $\sim 120-\mu \mathrm{m}$ trace thickness and a $\sim 2-\mathrm{mm}$ height. The mask was mounted on a 1.5 -mm-thick G-10 frame, which was later mounted inside the stainless-steel gas chamber, replacing the drift-mesh, $3 \mathrm{~mm}$ above the photocathode (see Fig. 1). The image was recorded irradiating $\sim 150 \mathrm{~mm}^{2}$ of the mask with the UV lamp at a distance of $\sim 25 \mathrm{~cm}$. Letters separated by $200-300 \mu \mathrm{m}$ are well resolved, demonstrating the good spatial resolution of the photodetector. Note that this on-line image was not cleaned from its background by any image-manipulation software.

a

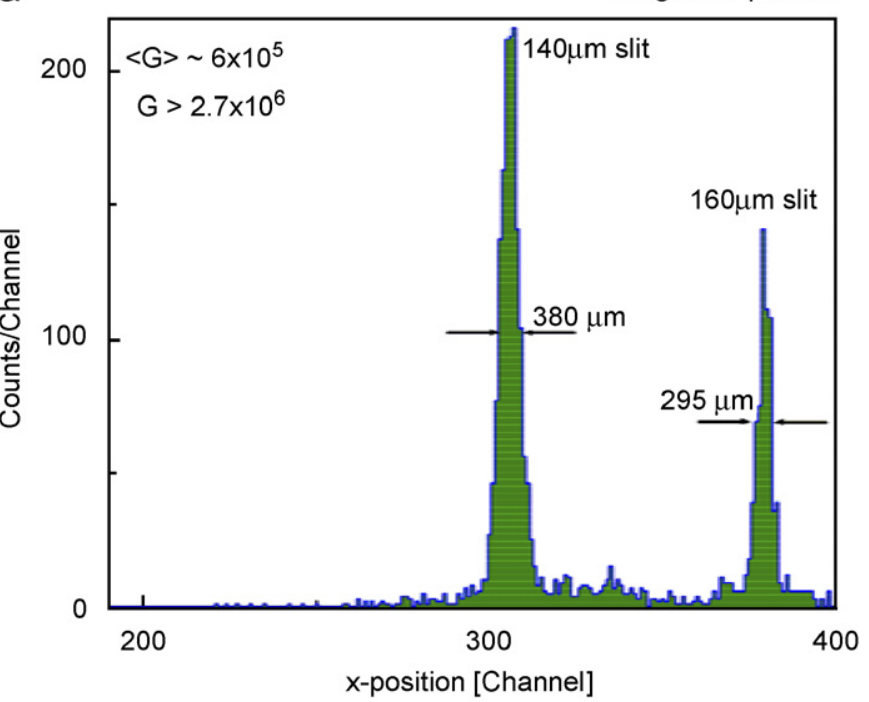

b

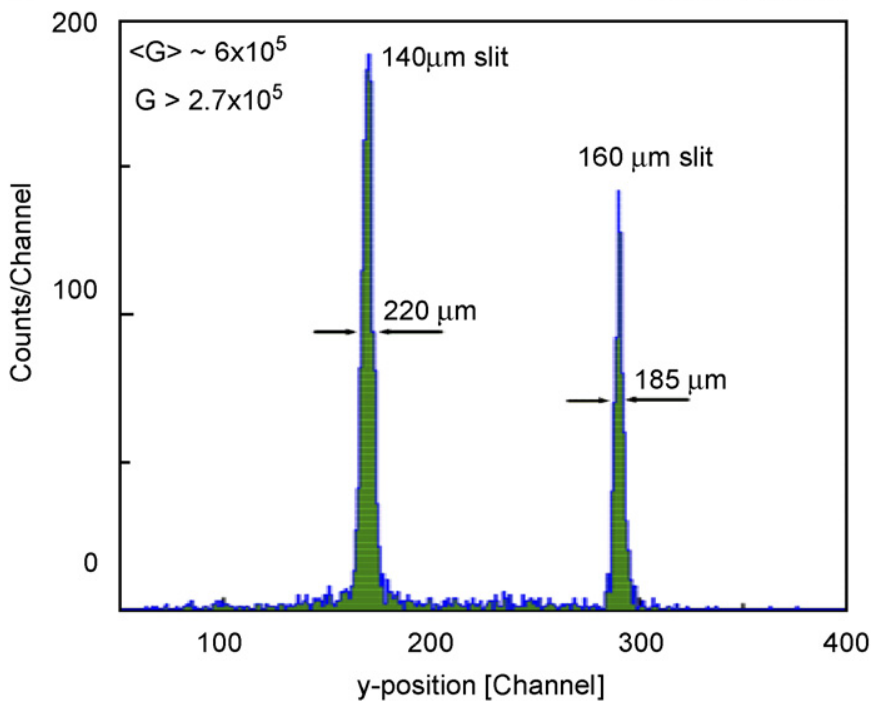

Fig. 3. Projections of the single-photon images of the 140- and $160-\mu \mathrm{m}$ width slits, obtained for an average gain of $\sim 6 \times 10^{5}$ and a threshold gain of $\sim 2.7 \times 10^{6}$ : (a) slits parallel and (b) slits perpendicular to the strips (S) of the $\mathrm{W} \& \mathrm{~S}$ readout. 


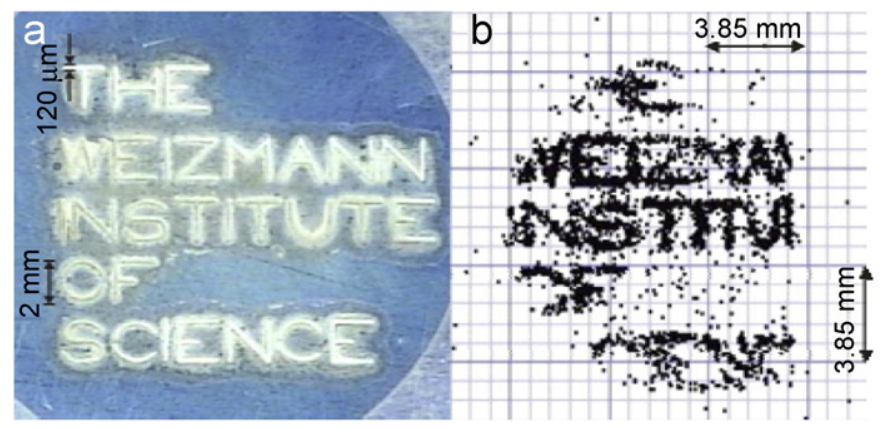

Fig. 4. A metal mask with the institute's name inscribed (a) and the respective 2-D single-photon image (b), recorded with an average gain of $\sim 5 \times 10^{5}$ and a threshold gain of $\sim 3.5 \times 10^{6}$.

\section{Summary}

We demonstrated the feasibility of a simple, low-cost GPM for single-photon imaging, with sub-millimeter resolution. It consists of a 4-GEM cascade with a reflective CsI-photocathode, coupled to a W\&S anode through a resistive electrode. Spatial resolutions of $\sim 150$ and $\sim 250 \mu \mathrm{m}$ (FWHM) for the $y$ - and $x$-positions, respectively, were obtained, using avalanche gains $>10^{6}$. These results are somewhat worse that the $\sim 125 \mu \mathrm{m}$ (FWHM) reported for a 3-GEM-based photodetector, coupled to a highdensity electronic readout through a $200-\mu \mathrm{m}$ pitch parallelstrip electrode [15]. In addition, the W\&S anode does not allow the reconstruction of double- and multi-hit events.
Complementary results of these studies will be reported in another article [16].

\section{Acknowledgments}

We acknowledge project POCI/FP/63414/05 from FCT, Portugal, and FEDER. J.M. Maia acknowledges the fellowship SFRH/BD $/ 9064 / 02$, FCT. A. Breskin is the W.P. Reuther Professor of Research in peaceful use of Atomic Energy.

\section{References}

[1] A. Breskin, et al., Nucl. Instr. and Meth. A 478 (2002) 225.

[2] F. Sauli, Nucl. Instr. and Meth. A 386 (1997) 531.

[3] D. Mörmann, et al., Nucl. Instr. and Meth. A 530 (2004) 258.

[4] R. Chechik, et al., IEEE Trans. Nucl. Sci. NS-51 (2004) 2097.

[5] D. Mörmann, et al., Nucl. Instr. and Meth. A 516 (2004) 315.

[6] D. Mörmann, et al., Nucl. Instr. and Meth. A 504 (2003) 93.

[7] A. Bressan, et al., Nucl. Instr. and Meth. A 425 (1999) 254.

[8] C. Altunbas, et al., Nucl. Instr. and Meth. A 490 (2002) 177.

[9] G.P. Guedes, et al., Nucl. Instr. and Meth. A 513 (2003) 473.

[10] V. Dangendorf, et al., Nucl. Instr. and Meth. A 542 (2005) 197.

[11] O.H.W. Siegmund, et al., IEEE Trans. Nucl. Sci. NS-33 (1) (1986) 724.

[12] O. Jagutzki, et al., Nucl. Instr. and Meth. A 477 (2002) 256.

[13] A. Buzulutskov, et al., Nucl. Instr. and Meth. A 443 (2000) 164.

[14] H. Klein, et al., Nucl. Instr. and Meth. A 193 (1982) 635.

[15] T. Meinschad, et al., Nucl. Instr. and Meth. A 535 (2004) 324.

[16] J.M. Maia, et al., Journal of Instrumentation (2007), submitted for publication. 